\title{
Interfacing Whispering Gallery Mode Optical Microresonator Biosensors with the Plant Defense Elicitor Chitin
}

\author{
Jeremy L. Dahmen ${ }^{\mathrm{A}}$, Yongqiang Yang ${ }^{\mathrm{B}}$, C. Michael Greenlief ${ }^{\mathrm{C}}$, Gary Stacey ${ }^{\mathrm{A}}$, Heather K. Hunt ${ }^{\mathrm{B}}{ }^{*}$ \\ ${ }^{\text {A }}$ Divisions of Plant Sciences and Biochemistry, Christopher S. Bond Life Sciences Center, University of Missouri, Colum- \\ bia, Missouri, 65211

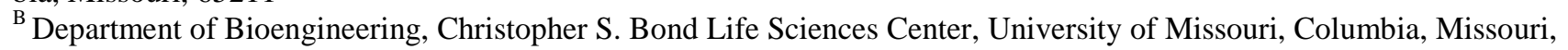 \\ 65211 \\ ${ }^{\mathrm{C}}$ Department of Chemistry, Christopher S. Bond Life Sciences Center, University of Missouri, Columbia, Missouri, 65211
}

* To whom correspondence should be addressed:

Department of Bioengineering, Christopher S. Bond Life Sciences Center, 240G, University of Missouri

1201 E. Rollins St., Columbia, MO 65211

hunthk@missouri.edu

573.882 .8202

keywords: chitin, n-acetylchitooligosaccharides, pathogen-associated molecular patterns, surface biochemistry, biosensors

\begin{abstract}
The biomaterial class of chitooligosaccharides (COs), commonly found in insects and fungi, is one of the most abundant on earth. Substantial evidence implicates COs in mediating a diverse array of plant cellular signaling events, including the induction of plant defense mechanisms against invading pests. However, the mechanisms for how recognition and mediation occur, including the kinetic parameters of binding interactions between COs and their plant recognition receptors, are not fully understood. Therefore, the creation of a platform capable of both interfacing with COs and plant cell receptors, and monitoring their interactions, would significantly advance our understanding of this plant defense elicitor. Recently, a labelfree, highly sensitive biosensor platform, based on Whispering Gallery Mode optical microresonators, has been developed to study such biomolecular interactions. Here, we demonstrate how this unique platform can be interfaced with COs, using simple carbohydrate chemistry, while maintaining sensitivity. The surface chemistry is demonstrated using X-ray photoelectron spectroscopy, fluorescence microscopy, optical profilometry, ellipsometry, and contact angle measurements. The bioconjugation layer on the platform is uniform with an average surface roughness of $1.25 \mathrm{~nm}$, and is active towards chitin recognition elements. Sensitivity measurements using standard quantitative cavity analysis techniques demonstrate that the bioconjugated platforms maintain the high sensitivity required to track binding interactions in this system. Due to their unique capabilities for label-free, high sensitivity biodetection when properly interfaced with the biomaterials of interest, this biosensor platform could provide the basis for a robust analytical technique that can probe the binding dynamics of chitin- plant cell receptors.
\end{abstract}

\section{Introduction}

A great number of agriculturally important crops suffer devastating losses every year from plant diseases. Of these diseases, fungal pathogens lead to the largest economic loss.[1] Plants depend upon their innate immune response to defend against these pathogens.[2-4] This immune response is triggered by two levels of microbial recognition: (1) the detection of pathogen (microbe) associated molecular patterns [P(M)AMPs] by pattern recognition receptors (PRRs) and (2) effector triggered immunity (ETI).[3] PAMPs are characterized both by being structurally conserved, or identical, across a wide array of microbes, and by not being produced by the host. Numerous studies have shown that PAMPs elicit an immune response in a number of agriculturally significant plants, such as soybean, rice, barley, and wheat.[5-7] PAMP signaling is also a key element in animal, including human, innate immunity systems.[8-9]

The ability to recognize various PAMPs enables plants to quickly defend against fungal, bacterial, and insect invaders. One of the most common PAMPs is chitin.[10] Chitin is comprised of polymers of N-acetylglucosamine (GlcNAc) residues linked together through a $\beta 1-4$ glycosidic linkage. Chitin is synthesized through the action of chitin synthases, which represent a very large gene family and are found not only in fungi and insects, but also in bacteria, protozoa, and vertebrates.[11]

In order for chitin to induce an immune response in plants, plasma membrane proteins, known as pattern recognition receptors (PRRs), must recognize and bind the chitin (Fig. 1).[12-14] Recently, the chitin receptors were characterized for both the genetic model plant (Arabidopsis thaliana), and rice (Oryza sativa).[15-17] Plasma membrane-bound, chitin-binding receptors are composed of a characteristic lysin (LysM) domain. This LysM motif is comprised of approximately 40 amino acids, which form a three dimensional $\beta \alpha \alpha \beta$ structure.[18-19]

Several different chitin elicitor binding proteins have been identified in several plant species, each with at least one LysM domain. For instance, in rice, a LysM-containing chitin oligosaccharide elicitor binding protein (CEBiP) was identified.[16] Oryza sativa (Os) CEBiP was found to contain two LysM domains, but to lack an intracellular, kinase domain found in many membrane bound receptors.[16] This led to the hypothesis that OsCeBiP requires a partner to form a receptor complex for downstream signaling.[16] Recently, it was shown in rice that a chitin elicitor receptor kinase (CERK1) is also involved in 
oligosaccharide perception, and forms a heterodimer with OsCEBiP.[13] In Arabidopsis thaliana, a chitin elicitor receptor kinase with three LysM domains and an intracellular serine/threonine kinase domain, was also identified (AtCERK1/LYK1).[15, 17]The X-ray crystal structure of AtCERK1 was recently solved, and the data indicate that a single AtCERK1 monomer can bind to chitotetroase, but does not trigger an intracellular response.[12] Apparently, homodimerization of AtCERK1, mediated by the binding higher molecular weight chitooligosaccharides (e.g., chitooctaose), is required to activate cellular signaling. It is well known that only chitooligossacharides (COs) of dp $>7$ have significant elicitor activity, at lease on rice and arabidopsis. However, a key issue with the AtCERK1 structure is the relatively low Kd values, in the $\mu \mathrm{M}$ range, while a variety of biological assays have shown that COs act at concentrations below $10 \mathrm{nM}$. Once COs bind to the specific receptors, downstream signaling will occur. Chitin binding to AtCERK1 results in activation of a mitogen-activated protein kinase (MAPK) cascade through phosphorylation (in Figure 1). This signaling cascade is responsible for activating the transcription of genes directly involved in pathogen defense, as well as transcription factors that will activate other defense-related genes (in Figure 1).[19, 21-22] For example, the WKRY transcription factor family, a class of DNA binding proteins found exclusively in plants, is involved in the activation of plant stress response genes.[23] Some fungi, including Cladosporiumfulvum (which causes leaf mold of tomato)and Magnaportheoryzae (the rice blast pathogen) secrete effector proteins that specifically target chitin signaling, reducing the defense response.[24]

Although some aspects of the chitin receptor systems are reasonably understood, their fundamental interaction processes need to be studied using a broad range of biological, chemical, and physical approaches to further elucidate how they trigger immune responses.[20] Previous work with label-based affinity binding assays, isothermal titration calorimetry, and molecular methods have established techniques for determining if a plant cell receptor will interact with chitin, but these methods lack either or both the sensitivity or specificity required to further elucidate the kinetic parameters of their actual interactions.[20] An alternative approach, based on a robust, sensitive and specific biosensor, would allow more detailed studies of the kinetic parameters of these interactions, as well as structural data to explain the chitin receptors affinity for various COs.

Optical biosensors are non-destructive sample interrogation methods with an ability to perform extremely sensitive detection in liquid environments due to their high signal-to-noise ratios. Common, high sensitivity optical methods for biosensing include labeled immunoassays, such as the ELISA assays and fluorescent immunoassays. Label-free optical biosensors, such as optical waveguides,[25-27] surface plasmon waveguides and resonators, [28-33] and WGM microresonators,[34] have demonstrated high sensitivity detection in multi-component systems when paired with an appropriate recognition element. These types of highly sensitive optical sensors have demonstrated $\mu \mathrm{M}$ to $\mathrm{nM}$ thresholds of sensitivity, and in the case of WGM microresonators, shown in Figure 2, are capable of detection down to the single nanoparticle or single virus level.[3538]

Of these, the surface plasmon resonance (SPR) system has been widely utilized to determine kinetic parameters of biological ligand-receptor interactions.[39-43] Recently, the WGM microresonators have also been used to study the binding kinetics of biomolecular interactions. For example, Soteropulos et al. (2011) determined the kinetic parameters of biotin-streptavidin binding using silica microsphere WGM microresonators.[44] Therefore, this highly sensitive biosensor platform has the potential to be very useful in defining kinetic parameters for protein-ligand complexes.[45-47]

However, for all the platforms mentioned, the primary challenge for tracking binding interactions remains pairing the high sensitivity biosensors with recognition elements that allow the sensor to interface with a specific biologically significant molecule, thereby reducing the risks of false positives and improving the overall device performance in multi-component systems.[48-49] In particular, interfacing semiconductor-type devices with biomolecules such as chitin or chitin receptors represents a significant challenge. Fortunately, there are a few studies on conjugating polymers to inorganic surfaces that could be well-suited to the chitin system.[50] These early functionalization techniques could be applied to these biosensor platforms, particularly for those platforms, like the WGM microresonators, that are fabricated from silica-based material systems. To do this, we must address three primary issues common to translating bioconjugation techniques to 3D devices: 1) biosensor surfaces tend to be easily damaged by typical wet chemistry techniques, resulting in a decrease in device performance; 2) standard protocols for heterogeneous chemistries, such as dispersed particles in solution, must be adapted to microscale devices so that the devices can be evenly functionalized across their 3D surfaces; and 3) plant biochemical reactions and typical bioconjugation strategies must be merged. From a device perspective, any post-fabrication modification that damages the surface, leaves residue on the surface, or results in a non-uniform surface coating, will negatively impact the device performance. This is due to the nature of the geometric optics that gives rise to the WGMs of the devices.

Here, we present a simple method to interface the important fungal cell wall constituent, chitin, to the surface of 3D, silicabased, microspherical, WGM microresonators, enabling the creation of a platform that can interact with identified, plant chitin receptors. This method will greatly expand our ability to probe plant signaling mechanisms via biosensors. Moreover, this report represents the first example of interfacing these types of optical biosensors with plant chemistry, and is the first time that chitin has been successfully, directly, bioconjugated to a sensor surface. 


\section{Materials and Methods}

In order to properly interface the biosensor platform with the chitin-chitin receptor system, the chitin must successfully be bioconjugated to the surface. Here, we base our initial approach on early literature describing acid-catalyzed ring-opening reactions, in combination with surface chemistry approaches commonly utilized in on-chip chemical processing.[50-51] Scheme 1 demonstrates the overall approach, which consists of three main steps: (i) surface hydroxylation, (ii) amination, and finally (iii) chitin conjugation via a ring-opening reaction. It is important to note that the chitin used throughout this work is actually a mixture of various COs, due to the high expense of purified octomeric chitin, and that the final addition of the chitin to the surface will yield an attached molecule with one less CO-mer than before, due to the ring-opening reaction.

\section{Device fabrication}

The silica microspheres used in this research were fabricated by gravimetric melting a tip of a stripped, single mode optical fiber (F-SE-OPT, Newport) with a $25 \mathrm{~W} \mathrm{CO} 2$ laser (48-2KAM, Synrad).[52] The microsphere was formed by surface tension during gravimetric melting, resulting in silica microspheres $80-100 \mu \mathrm{m}$ in diameter with an atomically smooth surface. After fabrication, the microspheres were placed in a microsphere holder and stored in a desiccator until use (see Supporting Information for microsphere holder description).[53]

\section{Device bioconjugation}

All chemicals and solvents used were certified ACS grade, except as otherwise indicated, and used as received without further purification. In order to successfully bioconjugate the microspheres without damaging the fragile glass surface, which would destroy the underlying device sensitivity during the hydroxylation and amination process, the microspheres were held within the microsphere holder. The bioconjugation reaction and subsequent washing steps were performed inside a lab-made reaction vessel (see Supporting Information for microsphere holder and reaction vessel).

First, the silica surface of the microspheres was terminated with hydroxyl groups via an oxygen plasma etch at $200 \mathrm{mTorr}$ pressure, $120 \mathrm{~W}$ output power for 5 minutes (Plasma Cleaner PE-50 Benchtop, Plasma Etch).[54-55]As demonstrated in the literature, this method of preparing the surface both removes surface contamination and results in surface silanol groups, without damage to the underlying device that more typical wet chemistry procedures, such as piranha or RCA etches, may yield.[55]

Second, the hydroxylated microspheres were aminated by grafting silane coupling agents to the silica surface using chemical vapor deposition.[55] Here, the hydroxylated microspheres, stored in the microsphere holder, were exposed to 3aminopropyltrimethoxysilane (APTMS, Sigma-Aldrich) vapor in a vacuum desiccator under 20 inch $\mathrm{Hg}$ vacuum for 15 minutes.[55]

Third, the COs were bound to the aminated microspheres via an acid-catalyzed ring opening reaction (see Supplemental Information). Sodium cyanoborohydride (Fisher) was used as the reducing agent to open the final ring of the chitin oligomers under an acidic condition by the addition of acetic acid (Aldrich). Here, we used chitin powder (shrimp shells, practical grade, $>95 \%$, Sigma-Aldrich), which consists of a variety of CO chain lengths. The chitin powder was suspended in the acidic solution, which was sonicated (Aquasonic 150D, VWR Scientific) for 15 minutes to improve dispersion. During the reaction with the microspheres, COs were reduced and covalently attached to the aminated silica surface as shown in Scheme 1, using a variety of reaction conditions to discover the optimal conditions that yielded a fully and uniformly bioconjugated surface, without damage to the underlying device (Table 1). For instance, high temperatures and stirring can induce thermal and mechanical stress in the devices, leading to structural damage of the silica surface, device decapitation, and sticky residue that creates areas of non-uniformity. Initial reaction conditions were chosen based on previously reported literature for the ring-opening reaction for chitin. [50] The chitin-bound microspheres were cooled to room temperature and washed with distilled water, methanol (Fisher), acetone (Fisher), heptanes (Aldrich) and acetone (Fisher) for 15 minutes, in that order, inside a clean reaction vessel on a tilt tray at room temperature. Finally, the microspheres were placed inside the microsphere holder, dried in an $80^{\circ} \mathrm{C}$ oven, and then stored in a desiccator.

\section{Device characterization}

Surface characterization of the conjugated devices was performed on both the devices themselves, as well as (100) silicon wafers (University Wafers), with a thin layer of silica native oxide on the surface, for those techniques that cannot address 3D surfaces. The silicon wafers were cut into $1 \times 1 \mathrm{~cm}$ pieces and then modified by following the same procedures as the microsphere bioconjugation.

X-ray photoelectron spectroscopy (XPS) was used to characterize the surface of the bioconjugated silicon wafers at each reaction step. XPS directly probes the surface composition by irradiating the surface of the silica wafers with X-rays (TA-10 twin X-ray source, VSW). The elements on the surface of the silicon wafers can be determined by an electron energy analyzer (15-255GAR, Physical Electronics) from the ejected electrons from the surface of $~ 10 \mathrm{~nm}$.[55] 
Fluorescence microscopy (LSM 510 META, Zeiss) was used to confirm the presence of chitin on the silica microsphere surfaces, and to compare reaction conditions in Table 1. Here, we evaluated both the amount of coverage and the quality of that coverage via fluorescent intensity measurements, with the best conditions yielding high intensities that were consistent across several areas of the imaged surface. The chitin-bound microspheres were compared to bare microspheres, to confirm that the fluorescent labeling was successful only on chitin-bound surfaces. Several post-labeling washing steps were done to minimize the possibility of non-specific adsorption of the labeling groups presenting false positives. In each case, the microspheres were incubated in $10 \mu \mathrm{g} / \mathrm{mL}$ lectin-fluorescein isothiocyanate (lectin-FITC, Sigma-Aldrich dye in PBS buffer (MP Biomedicals) for 30 minutes at room temperature. After lectin-FITC conjugation, the microspheres were washed and dried at room temperature.

The surface roughness of the conjugated silicon wafers was analyzed by optical profilometry (Wyko NT9100, Veeco). Both bare and bioconjugated silicon wafers were measured to compare the surface roughness before and after bioconjugation, since smooth surfaces are a key element of maintaining the performance of the WGM optical microresonator.[56]

Ellipsometry (VB400/HS-190, J.A. Woollam) was used to measure the thickness of the bioconjugated layer on the surface of the silicon wafers. The measurements were performed on both bare silicon wafers and bioconjugated silicon wafers. The reflectance was measured at multiple-angles-of-incidence $\left(65^{\circ}, 70^{\circ}\right.$ and $\left.75^{\circ}\right)$ using a scanning monochromator (HS-190, J.A. Woollam) within a wavelength range of 193 to $3200 \mathrm{~nm}$. The thickness of the bioconjugated layer was modeled using ellipsometry analysis software (WVASE32, J.A. Woollam).

Contact angle measurements (VCA Optima, AST Products) were performed on both silicon bare wafers and bioconjugated silicon wafers. The contact angles were automatically calculated by AutoFAST contact angle calculation software (AST Products).

\section{Quality factor study}

A quality factor study was used to analytically characterize the impact of the bioconjugation reactions on the device performance by measuring the resulting changes in optical loss. Here, we tested both the as-fabricated (bare, prior to hydroxylation) and chitin-bound microspheres (using only microspheres conjugated using the optimal reaction conditions) to determine the impact of the bioconjugation protocols on the device performance. Our key metric for success was to maintain the $\mathrm{Q}$ factor a bove $10^{6}$ post-conjugation, as this has been shown to be the cutoff for performing single virus detection.[57] Moreover, the higher the quality factor, the lower the limit of detection that is possible with the device for a given analyte. In this study, we used standard cavity characterization techniques to evaluate the $\mathrm{Q}$ factors of several devices. A tunable laser (TLB6700, Newport), coupled to a tapered optical fiber (F-SE-OPT, Newport), was used to couple ultra-narrow linewidth $(\sim 500 \mathrm{kHz})$ light in the wavelength range of 760-780 $\mathrm{nm}$ to the microspheres.[58] As the optical field exits the device, a photodetector (PDA36A, Thorlabs) is used to measure the transmitted optical field (Figure 3). We did not test the Q factor after reaction steps (i) and (ii) in Scheme 1, as our previous work confirms that these steps do not greatly impact the device sensitivity.[46, 54-55]

\section{Results}

\section{Surface chemistry}

With the addition of COs, we observed increases in nitrogen and carbon compared to the control wafers in the XPS spectra resulting from several different reaction conditions (Figure 4). From these data, we determined that the bioconjugation was optimally successful at $80^{\circ} \mathrm{C}$ for $1 \mathrm{~h}$. Other reaction times and temperatures yielded poor results from both a surface chemistry and a device damage perspective. The optimized reaction conditions were chosen for all future studies, including fluorescence microscopy and device characterization. We found it essential not to use standard wet chemistry techniques, such as piranha or RCA etches for the hydroxylation step of the syntheses, as this led to a substantial amount of residue and surface damage. Moreover, the number and type of washes at the end of the reaction were required to completely remove any debris from the surface. The primary challenge with these steps was preventing surface damage, which was easily minimized by using the custom microsphere holder and reaction vessel.

Fluorescent micrographs show that, using the optimal reaction conditions determined via XPS, chitin was successfully and uniformly bound to the aminated silica microspheres (Figure 5). Analysis of the bioconjugated microspheres showed significant fluorescence compared to the control (bare) microspheres, indicating that the microspheres were successfully bioconjugated with chitin. More importantly, these data showed that the bioconjugation protocols yielded a smooth, uniform surface without damaging the underlying device. These results were also used to optimize the washing steps post-modification to the conditions stated in the experimental section.

Optical profilometry test results, shown in Figure 6, on the chitin-bound silicon wafers showed that the surface of the wafers is relatively uniform, with an average surface roughness of $1.25 \mathrm{~nm}(\sigma, 0.28 \mathrm{~nm})$. 
After bioconjugation, the ellipsometry test results on the chitin-bound silicon wafers showed that the chitin layer of the wafer is $9.98 \mathrm{~nm}(\sigma, 3.36 \mathrm{~nm})$, which suggests a monolayer of chitin polymers was bioconjugated onto the silica surface. Compared to the bare silicon wafer surface, the chitin bound surfaces have a relatively higher standard deviation, which could be explained due to the monolayers on the silicon wafer surface resulting from a mixture of different lengths of COs.

Contact Angle Measurement showed that the contact angle of the chitin bound silicon wafer was slightly higher than the bare wafer. After bioconjugation, the devices' surfaces still showed hydrophilicity, which is necessary to interact with the chitin receptors in an aqueous environment. The mean of the contact angle (right and left angle) for bare wafer is $67.84^{\circ}\left(\sigma, 0.99^{\circ}\right)$. The mean of contact angle (right and left angle) for chitin bound wafer is $74.15^{\circ}\left(\sigma, 6.99^{\circ}\right)$. (See Supporting Information for contact angle measurements details).

\section{Platform Sensitivity}

The as-fabricated (bare) and chitin biocongugated microspheres were tested to determine the effects of chitin conjugation on the sensitivity of the device at 765-780 $\mathrm{nm}$. This wavelength range was chosen because the absorption of light is lower in water-based solutions (such as serum and buffer)comparing to the longer wavelengths (infrared spectrum).[59]Therefore, the devices could have a better performance at these wavelengths in future binding kinetics studies. In this case, we required the maintenance of the $\mathrm{Q}$ factor above $10^{6}$ after chitin was conjugated onto the surface of the microspheres. In Figure 7 , all of the bioconjugated microspheres show $\mathrm{Q}$ factors higher than $10^{6}$, although they have slightly lower $\mathrm{Q}$ factors than before bioconjugation. In this case, the data suggest that the slightly higher roughness of the coating, as well as the absorption of the chitin molecules themselves in this wavelength regime could account for this reduction in performance. Overall, however, the surface and resonator analysis indicates that this method of interfacing chitin with silica-based devices was successful.

\section{Discussion}

WGM microresonators, as biosensors, are highly sensitive to changes in the environment at the fM and potentially aM levels.[60] WGM microresonators can efficiently confine and circulate light within the microresonator periphery at specific resonant frequencies. Due to their unique geometric optics and inherent signal amplification, WGM microresonators can achieve ultra-low detection limits.[37, 61-64] This is because the WGM microresonators' optical field extends slightly into the surrounding environment through evanescence.[42,65]

The primary gauge of WGM microresonator quality is the device's quality factor, or Q factor, which describes the photon lifetime in the cavity and is directly related to its sensitivity. The photon lifetime $\left(\tau_{0}\right)$ in the cavity is dependent on the overall optical loss of the device. For example, a high-Q device (Q > 1.0E+08) has a high photon lifetime (> $100 \mathrm{~ns})$; a single photon in such a device will circulate, and potentially interact, over 100,000 times.[65] In comparison, planar evanescent sensors, like waveguide sensors, typically allow input photons to interact only once with the environment.

Using high-Q resonators, the interaction of molecules with the optical field can be detected via shifts in the resonance frequency of the resonator. This shift is a result of a change in the effective refractive index of the optical field, which itself can be due to small changes in diameter, optical loss, etc. In these devices, the high photon lifetime within the resonator imparts a high degree of sensitivity to the device, enabling the label-free detection of molecules, such as antigens, antibodies, and bacteria.[65]

We confirmed that chitin is conjugated to the microsphere biosensors with a monolayer of COs, without surface or structure damage, and without a significant, negative impact on the high sensitive of the devices. Although previous research has showed that CO derivatives can be spin-coated onto surfaces and then regenerated to a CO monolayer, with a very low roughness values on the surface[51], our method showed high promise in terms of maintaining the overall sensitivity of the biosensor. More significantly, it is suitable to functionalize the surface of the 3D devices, rather than just planar surfaces. Moreover, the bioconjugated COs layer could have a higher stability with regards to storage time, temperature, $\mathrm{pH}$ and other environmental changes during fabrication, storage, and detection.

Future studies will focus on using these newly developed chitin conjugated biosensors to study receptor-ligand interactions in the $\mathrm{CO}$ system. Based on our devices' label-free, high sensitivity and specificity, we can easily detect the bonding behaviors between chitin and its receptors. With these sensing test results, the kinetic parameters of their actual interactions between chitin and their recognition receptors in plants can be further elucidated.

\section{Conclusions}

A large number of agriculturally essential crops are negatively impacted every year by fungal plant diseases. A number of studies have shown that COs can elicit an immune response in a number of plants, such as rice, soybean, carrots, barley, tomato, wheat, and Arabidopsis.[6, 66-69] Previous studies allowed us to determine the plant cell receptors known to bind chitin. However, we have thus far lacked a technique that can accurately probe the kinetic parameters of these interactions at the appropriate concentration level. Due to their unique capabilities for label-free, high sensitivity and selectivity biodetection, the WGM optical microresonator platform could provide the basis for a robust analytical technique that can probe the bind- 
ing dynamics of chitin- plant cell receptors. Here, we demonstrate an important first step in this process: identifying the conditions at which such platforms can be successfully bioconjugated to interface with plant systems without negatively impacting the device performance. Moreover, the strategies given here can be widely applied to any type of silica-based, 3D structure, allowing many types of devices, beyond this specific biosensor platform, to probe these environments.

Developing a fundamental understanding of how elicitors, such as chitin, interact with plant cell receptors, will provide a more complete picture of plant disease resistance. Broadly speaking, such a detailed picture could lead to the construction of improved, disease-resistant crops, and the ability to help plants perceive and resist a broader range of pathogenic microbes and pests.

\section{Acknowledgements}

The authors acknowledge Charissa A. Nowak for the help with ellipsometry, contact angle and profilometry measurements, and Mark E. Anderson for the drawing of the schematic of experimental setup for testing microspheres' $Q$ factor. The authors also thank the MU International Center for Micro/Nano Systems \& Nanotechnology for use of their equipment and expertise. This work was supported by the Division of Chemical Sciences, Geosciences, and Biosciences, Office of Basic Energy Sciences of the U.S. Department of Energy through Grant DE-FG02-08ER15309 to GS.

\section{Abbreviations}

$\mathrm{CO}(\mathrm{s})$, Chitooligosaccharides; P(M)AMPs, pathogen (microbe) associated molecular patterns; PRRs, pattern recognition receptors; ETI, effector triggered immunity; GlcNAc, N-acetylglucosamine; CEBiP, elicitor binding protein; Os, oryzasativa; CERK1, chitin elicitor receptor kinase 1; MAPK, mitogen-activated protein kinase; ELISA, enzyme-linked immunosorbent assay; WGM, whispering gallery mode; SPR, surface plasmon resonance; APTMS, 3-aminopropyltrimethoxysilane; XPS, Xray photoelectron spectroscopy; Q factor, quality factor;; FITC, fluorescein isothiocyanate;. 


\section{References}

[1] A.Y. Rossman, The impact of invasive fungi on agricultural ecosystems in the United States, Biological Invasions, 11 (2009) 97-107.

[2] C. Zipfel, Early molecular events in PAMP-triggered immunity, Current Opinion in Plant Biology, 12 (2009) 414-420.

[3] T. Boller and S.Y. He, Innate Immunity in Plants: An Arms Race Between Pattern Recognition Receptors in Plants and Effectors in Microbial Pathogens, Science, 324 (2009) 742-744.

[4] J.D.G. Jones and J.L. Dangl, The plant immune system, Nature, 444 (2006) 323-329.

[5] R.B. Day, M. Okada, Y. Ito, K. Tsukada, H. Zaghouani, N. Shibuya and G. Stacey, Binding site for chitin

oligosaccharides in the soybean plasma membrane, Plant Physiology, 126 (2001) 1162-1173.

[6] M. Okada, M. Matsumura, Y. Ito and N. Shibuya, High-affinity binding proteins for N-acetylchitooligosaccharide elicitor in the plasma membranes from wheat, barley and carrot cells: Conserved presence and correlation with the responsiveness to the elicitor, Plant and Cell Physiology, 43 (2002) 505-512.

[7] N. Shibuya, N. Ebisu, Y. Kamada, H. Kaku, J. Cohn and Y. Ito, Localization and binding characteristics of a high-affinity binding site for $\mathrm{N}$-acetylchitooligosaccharide elicitor in the plasma membrane from suspension-cultured rice cells suggest a role as a receptor for the elicitor signal at the cell surface, Plant and Cell Physiology, 37 (1996) 894-898.

[8] S. Akira, K. Takeda and T. Kaisho, Toll-like receptors: critical proteins linking innate and acquired immunity, Nat Immunol, 2 (2001) 675-680.

[9] T. Nürnberger, F. Brunner, B. Kemmerling and L. Piater, Innate immunity in plants and animals: striking similarities and obvious differences, Immunological Reviews, 198 (2004) 249-266.

[10] M.D. Lenardon, C.A. Munro and N.A.R. Gow, Chitin synthesis and fungal pathogenesis, Current Opinion in Microbiology, 13 (2010) 416-423.

[11] C.E. Semino, C.A. Specht, A. Raimondi and P.W. Robbins, Homologs of the Xenopus developmental gene DG42 are present in zebrafish and mouse and are involved in the synthesis of Nod-like chitin oligosaccharides during early embryogenesis, Proceedings of the National Academy of Sciences, 93 (1996) 4548-4553.

[12] T. Liu, Z. Liu, C. Song, Y. Hu, Z. Han, J. She, F. Fan, J. Wang, C. Jin, J. Chang, J.-M. Zhou and J. Chai, Chitin-Induced Dimerization Activates a Plant Immune Receptor, Science, 336 (2012) 1160-1164.

[13] T. Shimizu, T. Nakano, D. Takamizawa, Y. Desaki, N. Ishii-Minami, Y. Nishizawa, E. Minami, K. Okada, H. Yamane, H. Kaku and N. Shibuya, Two LysM receptor molecules, CEBiP and OsCERK1, cooperatively regulate chitin elicitor signaling in rice, The Plant Journal, 64 (2010) 204-214.

[14] L.P. Hamel and N. Beaudoin, Chitooligosaccharide sensing and downstream signaling: Contrasted outcomes in pathogenic and beneficial plant-microbe interactions, Planta, 232 (2010) 787-806.

[15] J. Wan, X.-C. Zhang, D. Neece, K.M. Ramonell, S. Clough, S.-y. Kim, M.G. Stacey and G. Stacey, A LysM ReceptorLike Kinase Plays a Critical Role in Chitin Signaling and Fungal Resistance in Arabidopsis, The Plant Cell Online, 20 (2008) 471-481.

[16] H. Kaku, Y. Nishizawa, N. Ishii-Minami, C. Akimoto-Tomiyama, N. Dohmae, K. Takio, E. Minami and N. Shibuya, Plant cells recognize chitin fragments for defense signaling through a plasma membrane receptor, Proceedings of the National Academy of Sciences of the United States of America, 103 (2006) 11086-11091.

[17] A. Miya, P. Albert, T. Shinya, Y. Desaki, K. Ichimura, K. Shirasu, Y. Narusaka, N. Kawakami, H. Kaku and N. Shibuya, CERK1, a LysM receptor kinase, is essential for chitin elicitor signaling in Arabidopsis, Proceedings of the National Academy of Sciences, 104 (2007) 19613-19618.

[18] A. Bateman and M. Bycroft, The structure of a LysM domain from E. coli membrane-bound lytic murein transglycosylase D (MltD), Journal of Molecular Biology, 299 (2000) 1113-1119.

[19] X.-C. Zhang, X. Wu, S. Findley, J. Wan, M. Libault, H.T. Nguyen, S.B. Cannon and G. Stacey, Molecular Evolution of Lysin Motif-Type Receptor-Like Kinases in Plants, Plant Physiology, 144 (2007) 623-636.

[20] J.L. Dahmen, G. Stacey and H.K. Hunt, Current and emerging analytical technologies for analyzing chitin-protein binding interactions, Reviews in Analytical Chemistry, (2013) 1-20.

[21] G. Stacey and N. Shibuya, Chitin recognition in rice and legumes, Plant and Soil, 194 (1997) 161-169.

[22] N. Shibuya and E. Minami, Oligosaccharide signalling for defence responses in plant, Physiological and Molecular Plant Pathology, 59 (2001) 223-233.

[23] J. Wan, S. Zhang and G. Stacey, Activation of a mitogen-activated protein kinase pathway in Arabidopsis by chitin, Molecular Plant Pathology, 5 (2004) 125-135.

[24] T.A. Mentlak, A. Kombrink, T. Shinya, L.S. Ryder, I. Otomo, H. Saitoh, R. Terauchi, Y. Nishizawa, N. Shibuya, B.P.H.J. Thomma and N.J. Talbot, Effector-Mediated Suppression of Chitin-Triggered Immunity by Magnaporthe oryzae Is Necessary for Rice Blast Disease, The Plant Cell Online, 24 (2012) 322-335.

[25] R. Horvath, H.C. Pedersen, N. Skivesen, D. Selmeczi and N.B. Larsen, Optical waveguide sensor for on-line monitoring of bacteria, Opt. Lett., 28 (2003) 1233-1235.

[26] P. Dumais, C.L. Callender, J.P. Noad and C.J. Ledderhof, Silica-on-silicon optical sensor based on integrated waveguides and microchannels, Ieee Photonics Technology Letters, 17 (2005) 441-443.

[27] S. Andras, N. Adanyi, I. Szekacs, K. Majer-Baranyi and S. Istvan, Optical waveguide light-mode spectroscopy immunosensors for environmental monitoring, Applied Optics, 48 (2009) B151-B158. 
[28] D.R. Shankaran, K.V.A. Gobi and N. Miura, Recent advancements in surface plasmon resonance immunosensors for detection of small molecules of biomedical, food and environmental interest, Sens. Actuator B-Chem., 121 (2007) 158-177. [29] F. Sundberg and R. Karlsson, Rapid detection and characterization of immune responses using label-free biacore immunoassays, Immunology, 120 (2007) 46-47.

[30] S. Kumbhat, D.R. Shankaran, S.J. Kim, K.V. Gobi, V. Joshi and N. Miura, Surface plasmon resonance biosensor for dopamine using D3 dopamine receptor as a biorecognition molecule, Biosens. Bioelectron., 23 (2007) 421-427.

[31] T. Nagel, E. Ehrentreich-Forster, M. Singh, K. Schmitt, A. Brandenburg, A. Berka and F.F. Bier, Direct detection of tuberculosis infection in blood serum using three optical label-free approaches, Sens. Actuator B-Chem., 129 (2008) 934940.

[32] K.A. Tetz, L. Pang and Y. Fainman, High-resolution surface plasmon resonance sensor based on linewidth-optimized nanohole array transmittance, Opt. Lett., 31 (2006) 1528-1530.

[33] H.M. Chen, L. Pang, A. Kher and Y. Fainman, Three-dimensional composite metallodielectric nanostructure for enhanced surface plasmon resonance sensing, Appl. Phys. Lett., 94 (2009) 0731171-0731173.

[34] H. Li and X. Fan, Characterization of sensing capability of optofluidic ring resonator biosensors, Appl. Phys. Lett., 97 (2010) 011105-011103.

[35] F. Vollmer, S. Arnold and D. Keng, Single virus detection from the reactive shift of a whispering gallery mode, Proceedings of the National Academy of Sciences of the United States of America, 105 (2008) 20701-20704.

[36] F. Vollmer, D. Braun, A. Libchaber, M. Khoshsima, I. Teraoka and S. Arnold, Protein detection by optical shift of a resonant microcavity, Appl. Phys. Lett., 80 (2002) 4057-4059.

[37] N.M. Hanumegowda, I.M. White, H. Oveys and X.D. Fan, Label-free protease sensors based on optical microsphere resonators, Sens. Lett., 3 (2005) 315-319.

[38] J. Zhu, S.K. Ozdemir, Y.-F. Xiao, L. Li, L. He, D.-R. Chen and L. Yang, On-chip single nanoparticle detection and sizing by mode splitting in an ultrahigh-Q microresonator, Nat Photon, 4 (2010) 122-122.

[39] R.L. Rich and D.G. Myszka, Grading the commercial optical biosensor literature-Class of 2008: 'The Mighty Binders', Journal of Molecular Recognition, 23 (2010) 1-64.

[40] H.K. Hunt and A.M. Armani, Label-free biological and chemical sensors, Nanoscale, 2 (2010) 1544-1559.

[41] A. Abbas, M.J. Linman and Q.A. Cheng, New trends in instrumental design for surface plasmon resonance-based biosensors, Biosens. Bioelectron., 26 (2011) 1815-1824.

[42] X.D. Fan, I.M. White, S.I. Shopoua, H.Y. Zhu, J.D. Suter and Y.Z. Sun, Sensitive optical biosensors for unlabeled targets: A review, Analytica Chimica Acta, 620 (2008) 8-26.

[43] S.C.B. Gopinath, Biosensing applications of surface plasmon resonance-based Biacore technology, Sensors and Actuators, B: Chemical, 150 (2010) 722-733.

[44] C.E. Soteropulos, H.K. Hunt and A.M. Armani, Determination of binding kinetics using whispering gallery mode microcavities, Appl. Phys. Lett., 99 (2011).

[45] F. Vollmer and S. Arnold, Whispering-gallery-mode biosensing: label-free detection down to single molecules, Nature methods, 5 (2008) 591-596.

[46] C.E. Soteropulos, H.K. Hunt and A.M. Armani, Determination of binding kinetics using whispering gallery mode microcavities, Applied physics letters, 99 (2011) 103703-103703-103703.

[47] V.S. Ilchenko and A.B. Matsko, Optical resonators with whispering-gallery modes-part II: applications, Selected Topics in Quantum Electronics, IEEE Journal of, 12 (2006) 15-32.

[48] D. Erickson, S. Mandal, A.H.J. Yang and B. Cordovez, Nanobiosensors: optofluidic, electrical and mechanical approaches to biomolecular detection at the nanoscale, Microfluidics and Nanofluidics, 4 (2008) 33-52.

[49] B. Hock, M. Seifert and K. Kramer, Engineering receptors and antibodies for biosensors, Biosens. Bioelectron., 17 (2002) 239-249.

[50] K. Loos, V. von Braunmühl, R. Stadler, K. Landfester and H.W. Spiess, Saccharide modified silica particles by enzymatic grafting, Macromolecular Rapid Communications, 18 (1997) 927-938.

[51] J.D. Kittle, C. Wang, C. Qian, Y. Zhang, M. Zhang, M. Roman, J.R. Morris, R.B. Moore and A.R. Esker, Ultrathin chitin films for nanocomposites and biosensors, Biomacromolecules, 13 (2012) 714-718.

[52] C.E. Soteropulos, K.M. Zurick, M.T. Bernards and H.K. Hunt, Tailoring the Protein Adsorption Properties of Whispering Gallery Mode Optical Biosensors, Langmuir, 28 (2012) 15743-15750.

[53] C.E. Soteropulos and H.K. Hunt, Attaching Biological Probes to Silica Optical Biosensors Using Silane Coupling Agents, J Vis Exp, (2012) e3866.

[54] H.K. Hunt and A.M. Armani, Recycling microcavity optical biosensors, Optics letters, 36 (2011) $1092-1094$.

[55] H.K. Hunt, C. Soteropulos and A.M. Armani, Bioconjugation strategies for microtoroidal optical resonators, Sensors, 10 (2010) 9317-9336.

[56] K.J. Vahala, Optical microcavities, Nature, 424 (2003) 839-846.

[57] F. Vollmer, S. Arnold and D. Keng, Single virus detection from the reactive shift of a whispering-gallery mode, Proceedings of the National Academy of Sciences, 105 (2008) 20701-20704.

[58] J. Knight, G. Cheung, F. Jacques and T. Birks, Phase-matched excitation of whispering-gallery-mode resonances by a fiber taper, Optics letters, 22 (1997) 1129-1131. 
[59] G.M. Hale and M.R. Querry, Optical constants of water in the 200-nm to 200- $\mu$ m wavelength region, Applied optics, 12 (1973) 555-563.

[60] I.M. White, N.M. Hanumegowda and X.D. Fan, Subfemtomole detection of small molecules with microsphere sensors, Opt. Lett., 30 (2005) 3189-3191.

[61] M.L. Gorodetsky, A.A. Savchenkov and V.S. Ilchenko, Ultimate Q of optical microsphere resonators, Opt. Lett., 21 (1996) 453-455.

[62] A.B. Matsko, A.A. Savchenkov, D. Strekalov, V.S. Ilchenko and L. Maleki, Review of Applications of WhisperingGallery Mode Resonators in Photonics and Nonlinear Optics, The Interplanetary Network Progress Report, 42-162 (2005).

[63] N.M. Hanumegowda, C.J. Stica, B.C. Patel, I. White and X.D. Fan, Refractometric sensors based on microsphere resonators, Appl. Phys. Lett., 87 (2005).

[64] I.M. White, H.Y. Zhu, J.D. Suter, N.M. Hanumegowda, H. Oveys, M. Zourob and X.D. Fan, Refractometric sensors for lab-on-a-chip based on optical ring resonators, IEEE Sens. J., 7 (2007) 28-35.

[65] A.M. Armani, R.P. Kulkarni, S.E. Fraser, R.C. Flagan and K.J. Vahala, Label-free, single-molecule detection with optical microcavities, Science, 317 (2007) 783-787.

[66] G. Felix, M. Regenass and T. Boller, Specific perception of subnanomolar concentrations of chitin fragments by tomato cells: induction of extracellular alkalinization, changes in protein phosphorylation, and establishment of a refractory state, The Plant Journal, 4 (1993) 307-316.

[67] N. Shibuya, H. Kaku, K. Kuchitsu and M.J. Maliarik, Identification of a novel high-affinity binding site for $<\mathrm{i}>\mathrm{N}</ \mathrm{i}>-$ acetylchitooligosaccharide elicitor in the membrane fraction from suspension-cultured rice cells, FEBS letters, 329 (1993) 75-78.

[68] B. Zhang, K. Ramonell, S. Somerville and G. Stacey, Characterization of early, chitin-induced gene expression in Arabidopsis, Molecular Plant-Microbe Interactions, 15 (2002) 963-970.

[69] Y. Ito, H. Kaku and N. Shibuya, Identification of a high-affinity binding protein for N-acetylchitooligosaccharide elicitor in the plasma membrane of suspension-cultured rice cells by affinity labeling, The Plant Journal, 12 (1997) 347-356. 


\section{Figures}

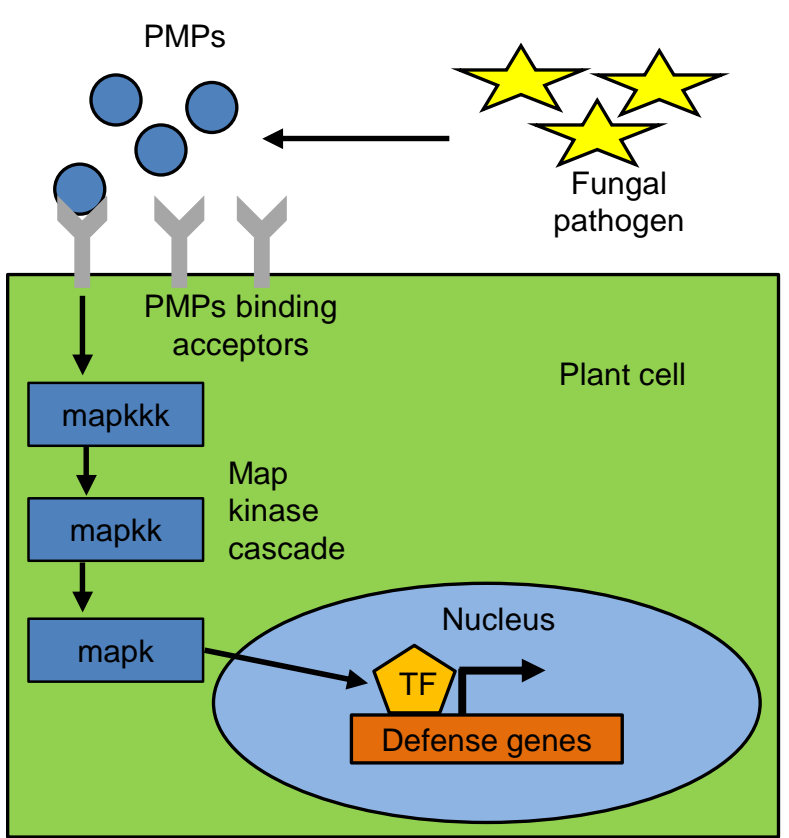

Figure 1. Model of the PAMP signaling pathway. Fungal pathogens release pathogen-associated molecular patterns (PAMPs), which bind to plant membrane associated pattern recognition receptors. The binding of PAMP leads to activation of the MAPK cascade, ultimately activating transcription factors, which mediate the expression of defense related genes. (Reprinted with permission from Dahmen et al. 2013, Ref [20])

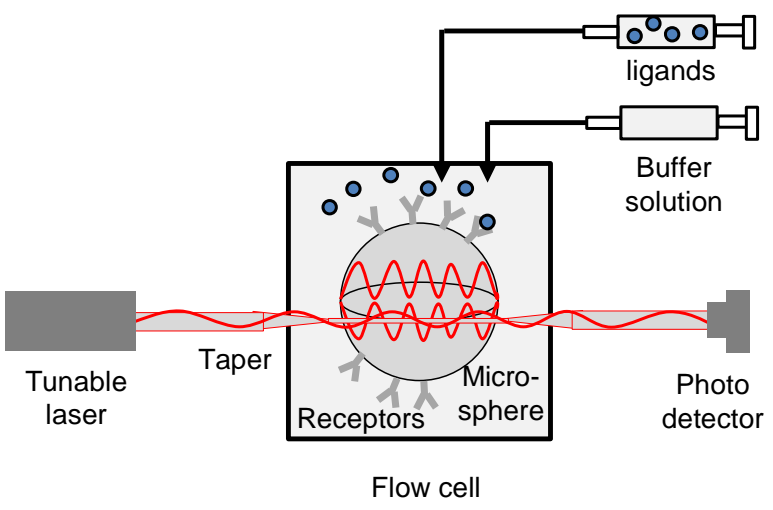

Figure 2. Schematic of a WGM optical biosensor to detect ligands in buffered solutions. In this WGM optical biosensor platform, the bioconjugated silica microsphere WGM optical microresonator is immersed in a buffer solution within a "microaquarium" style housing. Incoming laser light from a tunable laser is coupled into the microsphere by a tapered optical fiber. When analytes are injected into the "micro-aquarium," they are able to bind to the immobilized receptors on the bioconjugated device. This changes the optical properties of the circulating light in the device, leading to detectable shifts in the device's resonant frequency.

Table 1. The reaction conditions of chitin bioconjugation to (100) Si wafers show that higher temperatures and shorter reaction times produce favorable conjugation results.

\begin{tabular}{cccc}
\hline Trial & Temperature $\left({ }^{\circ} \mathrm{C}\right)$ & Time(h) & Results \\
& & & \\
1 & 50 & $>50$ & Poor \\
2 & 50 & 50 & Poor \\
3 & 50 & 70 & Poor \\
4 & 50 & $<70$ & Poor \\
5 & 80 & 1 & Excellent \\
6 & 80 & 12 & Good \\
\hline
\end{tabular}




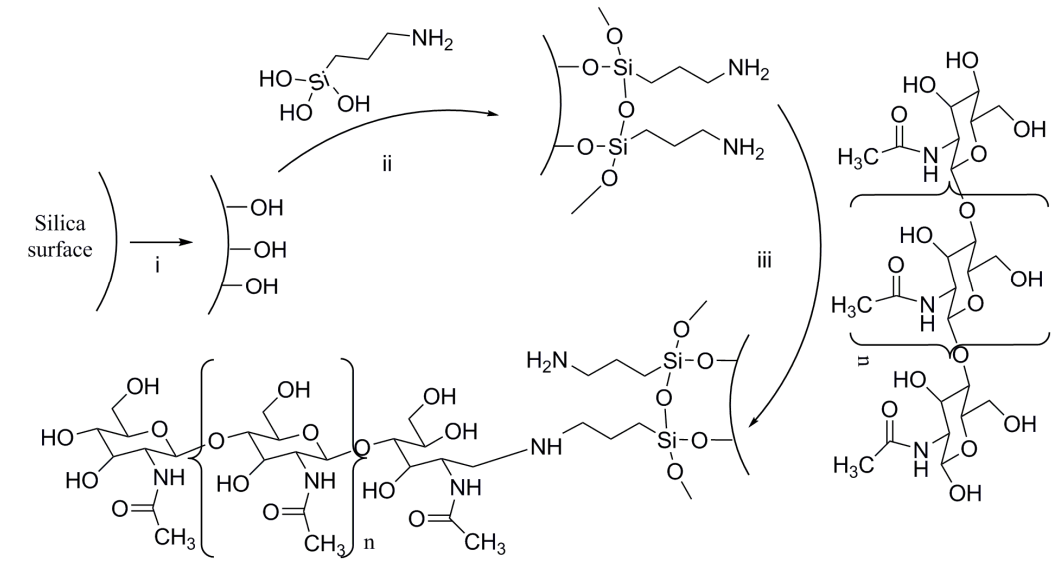

Scheme 1. Three step bioconjugation process to bind COs of various chitin chain lengths to the surface of the silica microspheres. In step (i), the microspheres are subjected to an oxygen plasma etch, creating hydroxyl groups on the silica surface; in step (ii), the hydroxylated microspheres are aminated via a chemical vapor deposition process with a silane coupling agent; and finally, in step (iii), the aminated microspheres are bioconjugated with COs via a ring opening reaction.

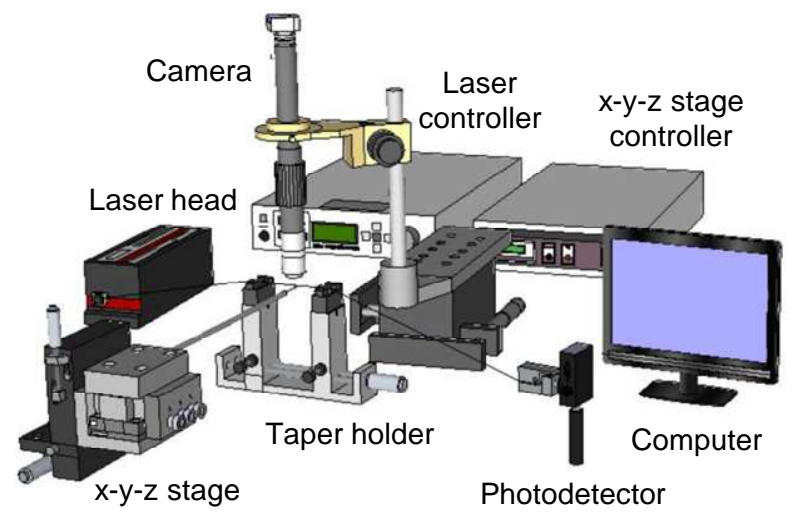

Figure 3. Schematic of the experimental setup for testing the silica microsphere WGM optical microresonator's performance. Key components are labeled.

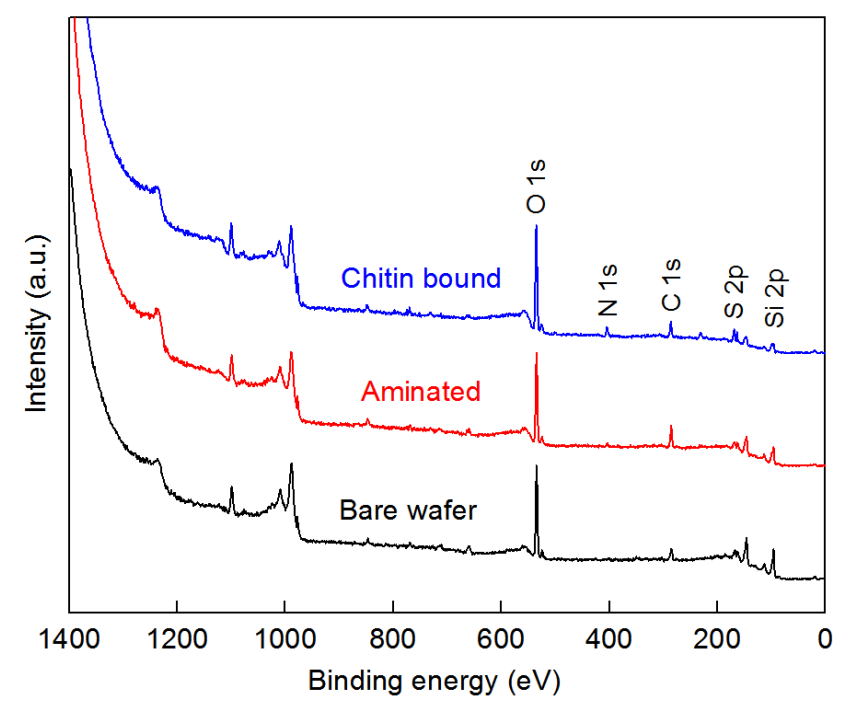

Figure 4. XPS data. The XPS data show that COs have been successfully biocojugated onto the surface of the silicon wafers, due to the increase in the presence of the nitrogen peak before and after step (iii) in Scheme 1. 


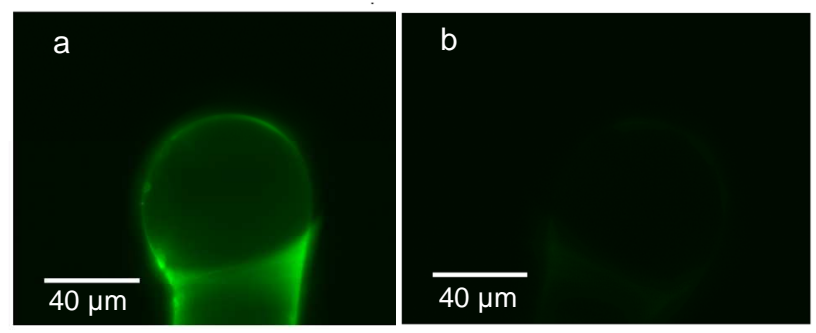

Figure 5. Fluorescent micrographs of microsphere exposed to lectin-FITC. (a) Bioconjugated microsphere. (b) Bare microsphere. Comparing the bare microsphere (b) to the bioconjugated microsphere (a), it is clear that the bioconjugation successfully yields a uniform surface coverage of chitin that is not the result of physical adsorption.
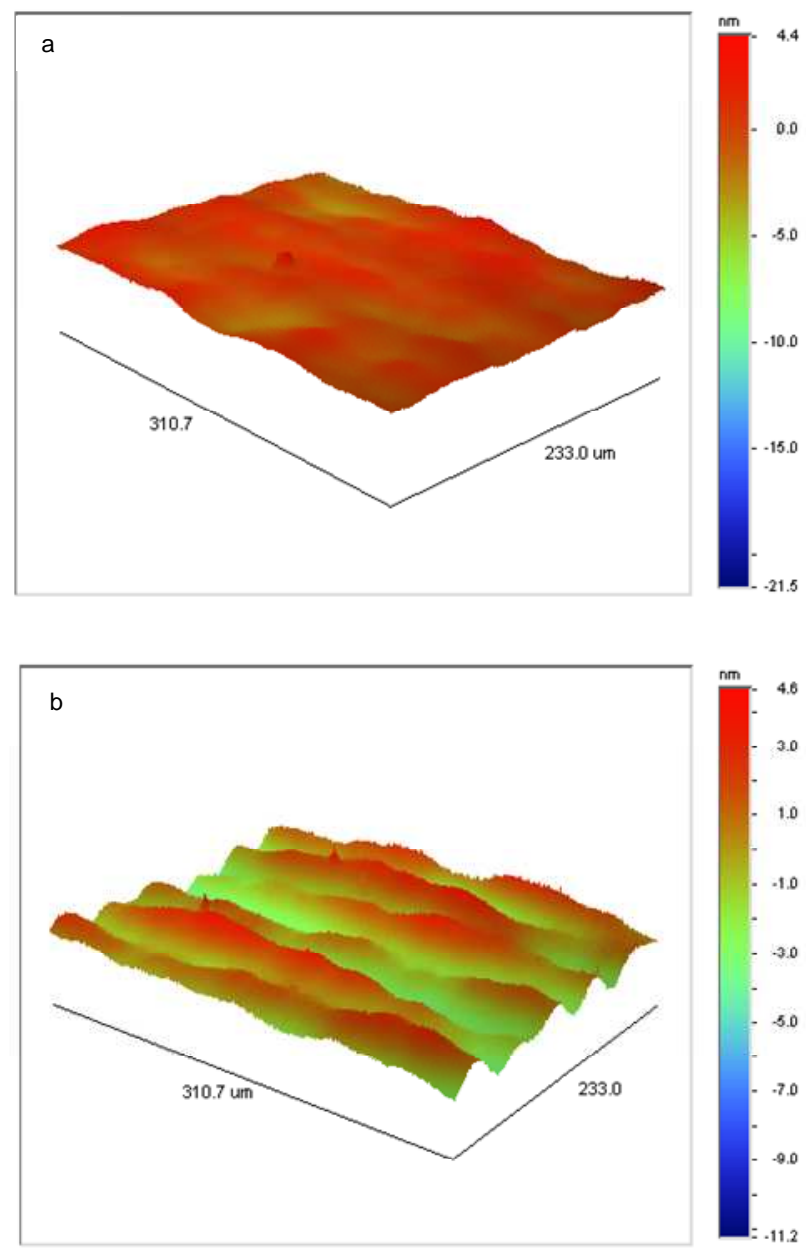

Figure 6. Optical profilometry measurements for bare wafer (a) and chitin bound wafer (b). The average surface roughness of the bare wafer (a) is $0.79 \mathrm{~nm}$ and RMS roughness is $1.05 \mathrm{~nm}$. The average surface roughness of the chitin bound wafer (b) is $1.18 \mathrm{~nm}$ and the RMS roughness is $1.48 \mathrm{~nm}$. The overall average is $1.25 \mathrm{~nm}$ and $\sigma$ is $0.28 \mathrm{~nm}$ for all the chitin-bound samples examined. 


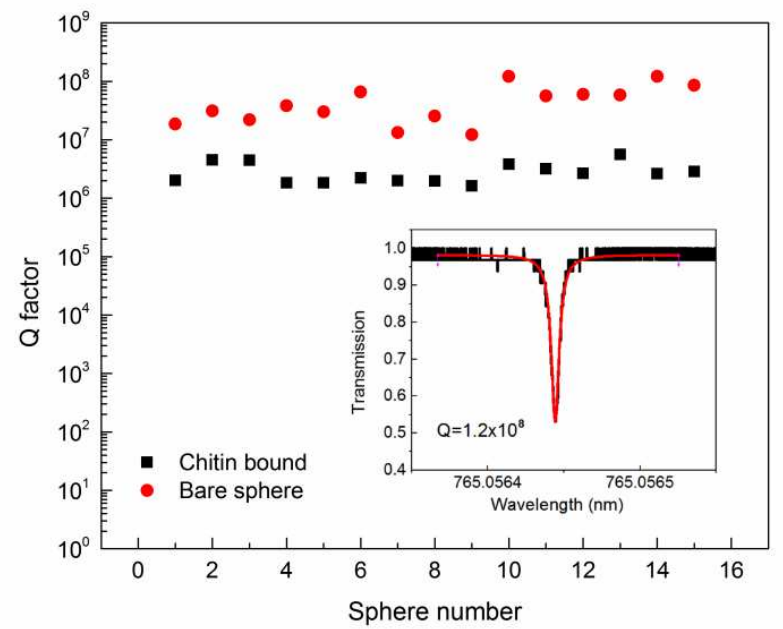

Figure 7. Quality factors of bare spheres and chitin bound spheres. The mean of $Q$ factors of 15 bare microspheres is $5.1 \mathrm{E}+07(\sigma, 3.6 \mathrm{E}+07)$. The mean $\mathrm{Q}$ factor after conjugation is $2.9 \mathrm{E}+06(\sigma, 1.2 \mathrm{E}+06)$. All functionalized microspheres present a $\mathrm{Q}$ factor of above 1.0E+06. Inset: Fitting a resonance peak (transmission dip, black) with a Lorentzian (red) to obtain the $\mathrm{Q}$ factor. 


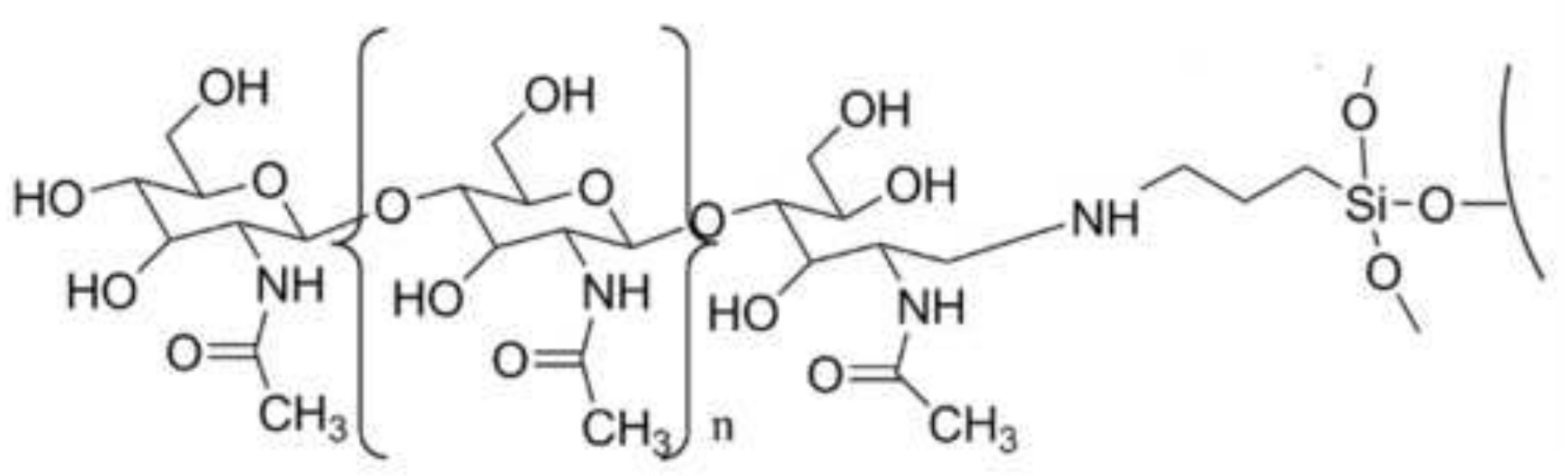

$40 \mu \mathrm{m}$ 